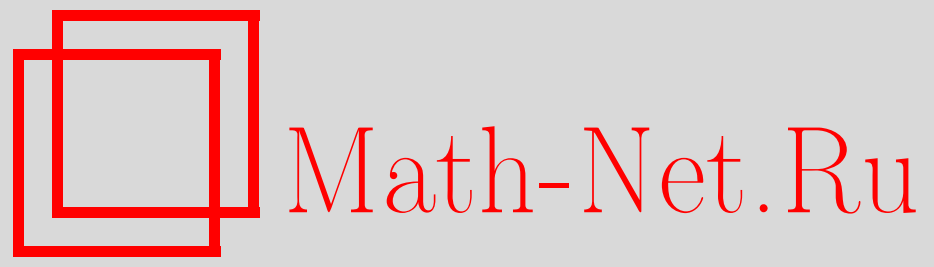

Д. А. Короткин, В. Б. Матвеев, О тэта-функциональных решениях системы Шлезингера и уравнения Эрнста, Функи. анализ и его прил., 2000, том 34, выпуск 4, 18-34

DOI: https://doi.org/10.4213/faa323

Использование Общероссийского математического портала MathNet.Ru подразумевает, что вы прочитали и согласны с пользовательским соглашением

http://www . mathnet.ru/rus/agreement

Параметры загрузки:

IP : 35.174 .16 .151

26 апреля 2023 г., 15:01:38

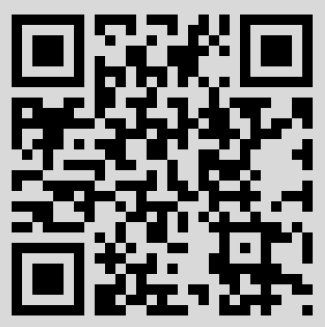




\title{
О тэта-функциональных решениях системы Шлезингера и уравнения Эрнста
}

\author{
(c) 2000. Д. А. Короткин, В. Б. МатвеЕв
}

\section{§1. Введение}

Алгебро-геометрический подход к решению солитонных уравнений возник из работ Новикова, Лакса, М. Каца, Итса, Матвеева, Дубровина, Мак-Кина, ван Мёрбеке и Кричевера, выполненных в 1974-1976 гг. Общей чертой большинства алгебро-геометрических решений является то, что динамика линеаризуется на якобиане фиксированной алгебраической кривой.

Концептуально новым явилось применение алгебро-геометрической идеологии к стационарному осесимметрическому уравнению Эйнштейна (уравнению Эрнста). Вложение этого уравнения в схему метода обратной задачи было инициировано работами $[1,2]$, где было найдено соответствующее лаксово представление ${ }^{1}$. В отличие от большинства интегрируемых систем эта пара параметризуется рациональной алгебраической кривой, зависящей от пространственно-временных переменных. Эта особенность влечет за собой коренные изменения в конструкции и качественных свойствах алгебро-геометрических решений, которые были впервые получены в 1988 г. в работах $[4,5]$. Эти решения строятся по гиперэллиптическим кривым, у которых две точки ветвления зависят от пространственновременных координат. Алгебро-геометрические решения уравнения Эрнста не обладают свойствами периодичности или почти периодичности, которые имеют место для всех уравнений типа КдФ. Более того, в случае уравнения Эрнста алгебро-геометрическая конструкция явно содержит функциональный параметр, что не имеет аналогов в $1+1$-интегрируемых системах типа КдФ. Широкий класс алгебро-геометрических решений уравнения Эрнста оказывается асимптотически плоским [6]. В вырожденном случае они включают весь класс многосолитонных решений Белинского и Захарова [1]. Простейшие эллиптические решения были изучены в работе [6]. Несмотря на многие интересные свойства, как оказалось, они содержат кольцевые сингулярности, что делает проблематичным их непосредственное физическое истолкование. Интересное физическое применение некоторого подсемейства алгебро-геометрических решений было предложено в работах $[7,8,10]$, которые посвящены исследованию граничной задачи, соответствующей бесконечно тонкому вращающемуся пылевому диску. В статье [10] была отмечена тесная связь теории алгебро-геометрических решений уравнения Эрнста с теорией скалярных задач Римана-Гильберта на гиперэллиптических кривых (см. обзор [11]). Сопоставление формул статей $[4]$ и $[7,8]$ было дано в работе [9], где

${ }^{1}$ Интересно отметить, что по существу та же пара Лакса фигурировала еще в работах Бьянки [3], посвященных специального вида конгруэнциям, называемым в настоящее время конгруэнциями Бьянки. 
было показано, что пылевой диск, описанный в $[7,8]$, является частным случаем решений рода 2 работы [4].

Данная статья имеет двоякую цель. Во-первых, мы устанавливаем связь между алгебро-геометрическими решениями уравнения Эрнста и алгебро-геометрическими решениями системы Шлезингера, построенными в недавней работе [13]. Это позволяет получить для алгебро-геометрических решений уравнения Эрнста простое выражение

$$
\mathscr{E}(\xi, \bar{\xi})=\frac{\Theta\left[\begin{array}{l}
\mathbf{r} \\
\mathbf{s}
\end{array}\right]\left(V(\xi)-V\left(\infty^{1}\right)\right)}{\Theta\left[\begin{array}{l}
\mathbf{r} \\
\mathbf{s}
\end{array}\right]\left(V(\xi)-V\left(\infty^{2}\right)\right)}
$$

в терминах тэта-функций с постоянными характеристиками $\mathbf{r}, \mathbf{s} \in \mathbb{C}^{g_{0}}$ и отображения Абеля, построенных по гиперэллиптической кривой $\mathscr{L}_{0}$ рода $g_{0}$ с двумя движущимися точками ветвления, заданной уравнением $\nu^{2}=(w-\xi)(w-\bar{\xi})$ $\times \prod_{j=1}^{2 g_{0}}\left(w-w_{j}\right)$. С помощью предельных переходов из формулы (1.1) можно получить все остальные алгебро-геометрические решения уравнения Эрнста. Вовторых, мы получаем явные формулы для всех коэффициентов метрики, соответствующих алгебро-геометрическим решениям уравнения Эрнста. Наиболее нетривиальным при этом является вычисление так называемого конформного фактора; для этого мы используем связь между конформным фактором и тау-функцией системы Шлезингера, найденную в [14], и формулу для тау-функции, полученную в работе [13]. Формула для конформного фактора, отвечающего решению (1.1), выглядит следующим образом:

$$
e^{2 k}=\frac{\Theta\left[\begin{array}{l}
\mathbf{r} \\
\mathbf{s}
\end{array}\right](0) \Theta\left[\begin{array}{l}
\mathbf{r} \\
\mathbf{s}
\end{array}\right]\left(\frac{1}{2} \mathbf{e}\right)}{\Theta(0) \Theta\left(\frac{1}{2} \mathbf{e}\right)}
$$

где $\mathbf{e} \equiv(1, \ldots, 1)$.

\section{§2. Система Шлезингера и стационарные осесимметричные уравнения Эйнштейна}

Рассмотрим линейное дифференциальное уравнение для функции $\Psi(\lambda) \in$ $S L(2, \mathbb{C})$

$$
\frac{d \Psi}{d \gamma}=\sum_{j=1}^{N} \frac{A_{j}}{\gamma-\gamma_{j}} \Psi
$$

где матрицы $A_{j} \in \operatorname{sl}(2, \mathbb{C})$ не зависят от $\gamma$. Наложим начальное условие $\Psi(\gamma=\infty)$ $=I$. Функция $\Psi(\gamma)$ однозначна на универсальной накрывающей $X$ римановой сферы с выколотыми точками $\mathbb{C} P^{1} \backslash\left\{\gamma_{1}, \ldots, \gamma_{N}\right\}$. Асимптотика функции $\Psi(\gamma)$ в окрестности сингулярностей $\gamma_{j}$ имеет (в ситуации общего положения) следующий вид: $\Psi(\gamma)=Q_{j}\left(I+O\left(\gamma-\gamma_{j}\right)\right)\left(\gamma-\gamma_{j}\right)^{T_{j}} C_{j}$, где $Q_{j}, C_{j} \in S L(2, \mathbb{C})$ и $T_{j}$ - бесследовая диагональная матрица. Матрицы $M_{j}=C_{j}^{-1} e^{2 \pi i T_{j}} C_{j}, j=1, \ldots, N$, называются матрицами монодромии. Условие независимости матриц $M_{j}$ от полюсов $\gamma_{j}$ называется условием изомонодромии; в ситуации общего положения (когда разность собственных значений ни одной из матриц $A_{j}$ не является целой) условие изомонодромии задает следующую зависимость $\Psi(\gamma)$ от $\gamma_{j}: \partial \Psi / \partial \gamma_{j}=-A_{j}\left(\gamma-\gamma_{j}\right)^{-1} \Psi$. Условия совместности этих уравнений с (2.1) эквивалентны системе [12] на вычеты $A_{j}$ :

$$
\frac{\partial A_{j}}{\partial \gamma_{i}}=\frac{\left[A_{i}, A_{j}\right]}{\gamma_{i}-\gamma_{j}}, \quad i \neq j, \quad \frac{\partial A_{i}}{\partial \gamma_{i}}=-\sum_{j \neq i} \frac{\left[A_{i}, A_{j}\right]}{\gamma_{i}-\gamma_{j}}
$$


Функция $\tau\left(\left\{\gamma_{j}\right\}\right)$, определенная уравнениями

$$
\frac{\partial}{\partial \gamma_{j}} \ln \tau=\sum_{k \neq j} \frac{\operatorname{tr} A_{j} A_{k}}{\gamma_{j}-\gamma_{k}}
$$

называется тау-функцией системы Шлезингера. Совместность уравнений (2.3) следует из системы Шлезингера.

Уравнения Эйнштейна для метрики вида

$$
d s^{2}=f^{-1}\left[e^{2 k}\left(d z^{2}+d \rho^{2}\right)+\rho^{2} d \varphi^{2}\right]-f(d t+F d \varphi)^{2},
$$

где все коэффициенты $f, k, F$ зависят только от $\rho$ и $z$, сводятся к уравнению Эрнста

$$
(\mathscr{E}+\overline{\mathscr{E}})\left(\mathscr{E}_{z z}+\rho^{-1} \mathscr{E}_{\rho}+\mathscr{E}_{\rho \rho}\right)=2\left(\mathscr{E}_{z}^{2}+\mathscr{E}_{\rho}^{2}\right) .
$$

Метрические коэффициенты могут быть найдены по комплекснозначному потенциалу Эрнста $\mathscr{E}(z, \rho)$ с помощью следующих уравнений:

$$
f=\operatorname{Re} \mathscr{E}, \quad \frac{\partial F}{\partial \xi}=2 \rho \frac{(\mathscr{E}-\overline{\mathscr{E}})_{\xi}}{(\mathscr{E}+\overline{\mathscr{E}})^{2}}, \quad \frac{\partial k}{\partial \xi}=2 i \rho \frac{\mathscr{E} \xi \overline{\mathscr{E}} \xi}{(\mathscr{E}+\overline{\mathscr{E}})^{2}},
$$

где $\xi=z+i \rho$. Уравнение Эрнста (2.5) является условием совместности следующей линейной системы:

$$
\Psi_{\xi}=\frac{G_{\xi} G^{-1}}{1-\gamma} \Psi, \quad \Psi_{\bar{\xi}}=\frac{G_{\bar{\xi}} G^{-1}}{1+\gamma} \Psi
$$

где

$$
\gamma=\frac{2}{\xi-\bar{\xi}}\left\{w-\frac{\xi+\bar{\xi}}{2}+\sqrt{(w-\xi)(w-\bar{\xi})}\right\}
$$

$w \in \mathbb{C}-$ спектральный параметр и

$$
G=\frac{1}{\mathscr{E}+\overline{\mathscr{E}}}\left(\begin{array}{cc}
2 & i(\mathscr{E}-\overline{\mathscr{E}}) \\
i(\mathscr{E}-\overline{\mathscr{E}}) & 2 \mathscr{E} \mathscr{E}
\end{array}\right)
$$

В терминах матрицы $G$ уравнение Эрнста может быть эквивалентным образом переписано в виде

$$
\left(\rho G_{\rho} G^{-1}\right)_{\rho}+\left(\rho G_{z} G^{-1}\right)_{z}=0 .
$$

Связь между системой Шлезингера и уравнением Эрнста была установлена в работе [14] и описывается следующий теоремой:

Теорема 2.1. Пусть матрицы $A_{j} \in \operatorname{sl}(2)$ удовлетворяют системам Шлезингера (2.2). Обозначим через $\Psi(\gamma)$ соответствующее решение системь (2.1). Пусть функция $\Psi(\gamma)$ удовлетворяет следующим дополнительным условиям:

$$
\begin{gathered}
\Psi^{t}(1 / \gamma) \Psi^{-1}(0) \Psi(\gamma)=I \\
\Psi(-\bar{\gamma})=\overline{\Psi(\gamma)} .
\end{gathered}
$$

Пусть, кроме того, $\gamma_{j}=\gamma\left(w_{j}, \xi, \bar{\xi}\right), w_{j} \in \mathbb{C}$ для всех $j$, где функция $\gamma(w, \xi, \bar{\xi})$ задается формулой (2.8). Тогда матрица

$$
G(\xi, \bar{\xi}) \equiv \Psi(\gamma=0, \xi, \bar{\xi})
$$


вещественна, симметрична и удовлетворяет уравнению Эрнста (2.10), а функция $\Psi$ удовлетворяет уравнениям (2.7). Коэффициент $e^{2 k}$ метрики (2.4) связан с $\tau$-функцией системы Шлезингера следующим образом:

$$
e^{2 k}=C \prod_{j=1}^{N}\left\{\frac{\partial \gamma_{j}}{\partial w_{j}}\right\}^{\operatorname{tr} A_{j}^{2} / 2} \tau,
$$

где $C$ - постоянная интегрирования.

\section{§3. Решения системы Шлезингера в терминах тэта-функций}

Пусть $N=2 g+2$. Рассмотрим гиперэллиптическую кривую $\mathscr{L}$ рода $g$, определенную уравнением

$$
y^{2}=\prod_{j=1}^{2 g+2}\left(\gamma-\gamma_{j}\right)
$$

и выберем базисные циклы $\left(a_{j}, b_{j}\right)$ на $\mathscr{L}$ в соответствии с рис. 1.

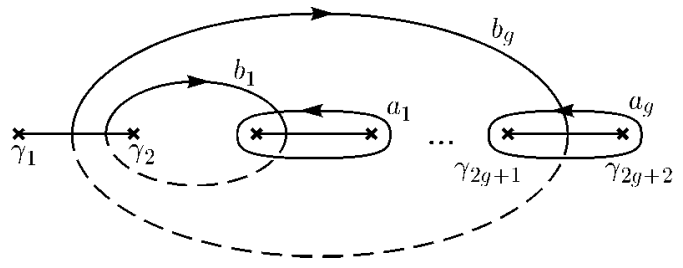

Pис. 1

Базисные голоморфные дифференциалы на $\mathscr{L}$ имеют вид $\gamma^{k-1} d \gamma / y, k=$ $1, \ldots, g$. Рассмотрим матрицы $a$ - и $b$-периодов этих дифференциалов:

$$
\mathscr{A}_{k j}=\oint_{a_{j}} \frac{\gamma^{k-1} d \gamma}{y}, \quad \mathscr{B}_{k j}=\oint_{b_{j}} \frac{\gamma^{k-1} d \gamma}{y} .
$$

Тогда голоморфные потенциалы $d U_{k}=\frac{1}{y} \sum_{j=1}^{g}\left(\mathscr{A}^{-1}\right)_{k j} \gamma^{j-1} d \gamma$ будут удовлетворять условиям нормировки $\oint_{a_{j}} d U_{k}=\delta_{j k}$. По матрицам $\mathscr{A}$ и $\mathscr{B}$ строится симметричная матрица $b$-периодов кривой $\mathscr{L}: \mathbf{B}=\mathscr{A}^{-1} \mathscr{B}$. Рассмотрим стандартную тэта-функцию с характеристиками $\Theta[\mathbf{q}](\mathbf{z} \mid \mathbf{B})\left(\mathbf{p}, \mathbf{q}, \mathbf{z} \in \mathbb{C}^{g}\right)$. Она обладает следующими свойствами периодичности:

$$
\begin{gathered}
\Theta\left[\begin{array}{l}
\mathbf{p} \\
\mathbf{q}
\end{array}\right]\left(\mathbf{z}+\mathbf{e}_{j}\right)=e^{2 \pi i p_{j}} \Theta\left[\begin{array}{l}
\mathbf{p} \\
\mathbf{q}
\end{array}\right](\mathbf{z}), \\
\Theta\left[\begin{array}{l}
\mathbf{p} \\
\mathbf{q}
\end{array}\right]\left(\mathbf{z}+\mathbf{B e}_{j}\right)=e^{-2 \pi i q_{j}} e^{-\pi i \mathbf{B}_{j j}-2 \pi i \mathbf{z}_{j}} \Theta\left[\begin{array}{l}
\mathbf{p} \\
\mathbf{q}
\end{array}\right](\mathbf{z}),
\end{gathered}
$$

где $\mathbf{e}_{j} \equiv(0, \ldots, 1, \ldots, 0)^{t}$ (1 стоит на $j$-м месте). Разрежем кривую $\mathscr{L}$ вдоль всех базисных циклов, начинающихся из любой фиксированной точки; получившийся фундаментальный многоугольник обозначим через $\widehat{\mathscr{L}}$. Мы будем также использовать следующее обозначение: $\left.W\right|_{Q} ^{P} \equiv \int_{Q}^{P} d W$, где $d W-$ произвольный мероморфный дифференциал на $\mathscr{L}$ и контур интегрирования лежит внутри $\widehat{\mathscr{L}}$ (если $d W$ имеет полюсы первого порядка, то этот интеграл зависит также 
от выбора контура интегрирования внутри $\widehat{\mathscr{L}}$ ). Через $\left.U\right|_{Q} ^{P}$ мы обозначим вектор с компонентами $\left.U_{j}\right|_{Q} ^{P}$. Вектор римановых констант, соответствующий нашему выбору начальной точки интегрирования, выглядит следующим образом [15]: $K=\frac{1}{2} \mathbf{B}\left(\mathbf{e}_{1}+\cdots+\mathbf{e}_{g}\right)+\frac{1}{2}\left(\mathbf{e}_{1}+2 \mathbf{e}_{2}+\cdots+g \mathbf{e}_{g}\right)$.

Векторы характеристик с компонентами $\mathbf{p} \in \mathbb{C}^{g} / 2 \mathbb{C}^{g}, \mathbf{q} \in \mathbb{C}^{g} / 2 \mathbb{C}^{g}$ называются полуцелыми; они находятся во взаимно однозначном соответствии с полупериодами $\mathbf{B p}+\mathbf{q}$. Если скалярное произведение $4\langle\mathbf{p}, \mathbf{q}\rangle$ нечетно, то нечетна и соответствующая тэта-функция как функция от $\mathbf{z}$ и вектор характеристик $\left[\begin{array}{l}\mathbf{p} \\ \mathbf{q}\end{array}\right]$ называется нечетным; в противном случае $\Theta[\mathbf{p}](\mathbf{q})$ четна и вектор характеристик $\left[\begin{array}{l}\mathbf{p} \\ \mathbf{q}\end{array}\right]$ называется четным. Нас будут в дальнейшем интересовать нечетные векторы характеристик, которые могут быть построены по произвольному набору $S=\left\{\gamma_{i_{1}}, \ldots, \gamma_{i_{g-1}}\right\}$ из $g-1$ несовпадающих точек ветвления. Нечетный полупериод, соответствующий набору $S$, задается формулой

$$
\mathbf{B} \mathbf{p}^{S}+\mathbf{q}^{S}=\left.\sum_{j=1}^{g-1} U\right|_{\gamma_{1}} ^{\gamma_{i_{j}}}-K,
$$

где $d U=\left(d U_{1}, \ldots, d U_{g}\right)^{t}$. Обозначим через $\Omega_{\gamma} \subset \mathbb{C}$ произвольную окрестность $\Omega_{\gamma} \subset \mathbb{C}$ бесконечной точки $\gamma=\infty$, такую, что $\Omega_{\gamma}$ не имеет пересечений с проекциями базисных циклов на $\gamma$-плоскость. Определим матричнозначную $2 \times 2$-функцию $\Phi(\gamma)$ в области $\Omega_{\gamma}$, лежащей на первом листе кривой $\mathscr{L}$, по формуле

$$
\Phi\left(\gamma \in \Omega_{\gamma}\right)=\left(\begin{array}{ll}
\varphi(\gamma) & \varphi\left(\gamma^{*}\right) \\
\psi(\gamma) & \psi\left(\gamma^{*}\right)
\end{array}\right)
$$

где функции $\varphi$ и $\psi$ определены внутри $\widehat{\mathscr{L}}$ формулами

$$
\begin{aligned}
& \varphi(\gamma)=\Theta\left[\begin{array}{l}
\mathbf{p} \\
\mathbf{q}
\end{array}\right]\left(\left.U\right|_{\gamma_{1}} ^{\gamma}+\left.U\right|_{\gamma_{1}} ^{\gamma_{\varphi}} \mid \mathbf{B}\right) \Theta[S]\left(\left.U\right|_{\gamma_{\varphi}} ^{\gamma} \mid \mathbf{B}\right), \\
& \psi(\gamma)=\Theta\left[\begin{array}{l}
\mathbf{p} \\
\mathbf{q}
\end{array}\right]\left(\left.U\right|_{\gamma_{1}} ^{\gamma}+\left.U\right|_{\gamma_{1}} ^{\gamma_{\psi}} \mid \mathbf{B}\right) \Theta[S]\left(\left.U\right|_{\gamma_{\psi}} ^{\gamma} \mid \mathbf{B}\right),
\end{aligned}
$$

$\gamma_{\varphi}$ и $\gamma_{\psi} \in \mathscr{L}-$ две произвольные (возможно, зависящие от $\left\{\gamma_{j}\right\}$ ) точки на $\mathscr{L}$, $\left[\begin{array}{l}\mathbf{p} \\ \mathbf{q}\end{array}\right]$ - произвольный вектор характеристик, не зависящих от $\left\{\gamma_{j}\right\}$, * - переставляющая листы инволюция на $\mathscr{L}, \Theta[S](\mathbf{z}) \equiv \Theta\left[\begin{array}{l}\mathbf{p}_{\mathbf{q}^{S}}^{S} \\ ]\end{array}\right](\mathbf{z})$ и нечетный вектор характеристик $\left[\begin{array}{l}\mathbf{p}^{S} \\ \mathbf{q}^{S}\end{array}\right]$ построен по произвольному набору $S$ из $g-1$ точек ветвления посредством формулы (3.4). Поскольку область $\Omega_{\gamma}$ не пересекается с проекциями базисных циклов на $\gamma$-плоскость, область $\Omega_{\gamma}^{*}$ не пересекается с границей фундаментального многоугольника $\widehat{\mathscr{L}}$, и, следовательно, функции $\varphi\left(\gamma^{*}\right)$ и $\psi\left(\gamma^{*}\right)$ из (3.5) определяются формулами (3.6) при $\gamma \in \Omega_{\gamma}$ единственным образом. Выберем теперь некоторый лист универсальной накрывающей $X$ и определим новую функцию $\Psi(\gamma)$ в области $\Omega_{\gamma}$, лежащей на этом листе, по формуле

$$
\Psi\left(\gamma \in \Omega_{\gamma}\right)=\sqrt{\frac{\operatorname{det} \Phi\left(\infty^{1}\right)}{\operatorname{det} \Phi(\gamma)}} \Phi^{-1}\left(\infty^{1}\right) \Phi(\gamma) .
$$

Аналитически продолжим функцию $\Psi(\gamma)$ на всю $X$. Функция $\Psi(\gamma)$ из (3.7) преобразуется следующим образом при обходе вокруг базисных циклов (обозначим через $T_{a_{j}}$ и $T_{b_{j}}$ соответствующие операторы аналитического продол- 
жения): $T_{a_{j}}[\Psi(\gamma)]=\Psi(\gamma) M_{a_{j}}, T_{b_{j}}[\Psi(\gamma)]=\Psi(\gamma) M_{b_{j}}, j=1, \ldots, 2 g_{0}-1$, где

$$
M_{a_{j}}=\left(\begin{array}{cc}
e^{2 \pi i p_{j}} & 0 \\
0 & e^{-2 \pi i p_{j}}
\end{array}\right), \quad M_{b_{j}}=\left(\begin{array}{cc}
e^{-2 \pi i q_{j}} & 0 \\
0 & e^{2 \pi i q_{j}}
\end{array}\right) .
$$

Теорема 3.1 ([13]). Обозначим через $(\mathbf{p}, \mathbf{q}), \mathbf{p}, \mathbf{q} \in \mathbb{C}^{g}$, произвольный набор из $2 g$ констант, таких, что вектор характеристик $\left[\begin{array}{l}\mathbf{p} \\ \mathbf{q}\end{array}\right]$ не является полуиельм. Tогда

(1) функиия $\Psi(Q \in X)$, заданная формулой (3.7), не зависит от $\gamma_{\varphi} u \gamma_{\psi} u$ удовлетворяет уравнению (2.1), причем вычеты $\left.A_{j} \equiv \operatorname{res}\right|_{\gamma=\gamma_{j}}\left\{\Psi_{\gamma} \Psi^{-1}\right\}$ удовлетворяют системе Шлезингера (2.2);

(2) монодромии функции $\Psi(\gamma)$ вокруг точек $\gamma_{j}$ имеют вид $M_{j}=\left(\begin{array}{cc}0 & -m_{j} \\ m_{j}^{-1} & 0\end{array}\right)$, где матричные элементы $m_{j}$ выражаются через р и q (см. [13]);

(3) тау-функция, соответствующцая этому решению системы Шлезингера, имеет вид

$$
\tau\left(\left\{\gamma_{j}\right\}\right)=[\operatorname{det} \mathscr{A}]^{-1 / 2} \prod_{j<k}\left(\gamma_{j}-\gamma_{k}\right)^{-1 / 8} \Theta\left[\begin{array}{l}
\mathbf{p} \\
\mathbf{q}
\end{array}\right](0 \mid \mathbf{B})
$$

\section{§4. Алгебро-геометрические решения уравнения Эрнста}

Теорема 2.1 позволяет получить решения уравнения Эрнста, исходя из решений системы Шлезингера, описанных выше. Необходимая дополнительная работа состоит в выборе параметров конструкции (т.е. точек $w_{j}$ и векторов $\mathbf{p}, \mathbf{q}$ ) таким образом, чтобы обеспечить выполнение уравнений (2.11) и (2.12). Для этого необходимо предположить, что кривая $\mathscr{L}$ инвариантна относительно голоморфной инволюции $\sigma$ и антиголоморфной инволюции $\mu$, действующих на каждом листе кривой $\mathscr{L}$ следующим образом: $\sigma: \gamma \rightarrow 1 / \gamma, \mu: \gamma \rightarrow-\bar{\gamma}$. Уравнения (2.11) и (2.12) совместны, только если кривая $\mathscr{L}$ имеет нечетный род, $g=2 g_{0}-1$. Мы пронумеруем точки ветвления $\gamma_{j}, j=1, \ldots, 4 g_{0}$, таким образом, что $\gamma_{j}=\gamma_{j+2 g_{0}}^{-1}$, $j=1, \ldots, 2 g_{0}$, и $\gamma_{j} \in i \mathbb{R}, j \leqslant 2 k, \gamma_{2 j+1}+\bar{\gamma}_{2 j+2}=0, j \geqslant 2 k+1$ для некоторого $k \leqslant g_{0}$. Рассмотрим два случая:

1. Кривая $\mathscr{L}$ является кривой неразделяющегося типа по отношению к антиинволюции $\mu$, т. е. $k \geqslant 1$. Тогда базисные циклы $\left(a_{j}, b_{j}\right), j=1, \ldots, 2 g_{0}-1$, на $\mathscr{L}$ могут быть выбраны так, как показано на рис. 2a).

2. Кривая $\mathscr{L}$ является кривой разделяющегося типа по отношению к антиинволюции $\mu$, т. е. $k=0$. Тогда базисные циклы могут быть выбраны, как показано на рис. $2 \mathrm{~b}$ ).

В обоих случаях базисные циклы преобразуются следующим образом под действием инволюции $\sigma$ :

$$
\begin{gathered}
\sigma\left(a_{1}\right)=-a_{1}, \quad \sigma\left(b_{1}\right)=-b_{1}, \\
\sigma\left(a_{j}\right)=a_{j+g_{0}-1}, \quad \sigma\left(b_{j}\right)=b_{j+g_{0}-1}, \quad 2 \leqslant j \leqslant g_{0} .
\end{gathered}
$$

Уравнение (2.11) налагает такие условия на монодромии $M_{a_{j}}$ и $M_{b_{j}}$, заданные формулами (3.8): $M_{a_{j}}^{t} M_{a_{j+g_{0}-1}}=I, M_{b_{j}}^{t} M_{b_{j+g_{0}-1}}=I, 2 \leqslant j \leqslant g_{0}$. (Уравнения $M_{a_{1}}^{t}=M_{a_{1}}$ и $M_{b_{1}}^{t}=M_{b_{1}}$, которые возникают при вычислении монодромий левой части условия (2.11) вокруг базисных циклов $a_{1}$ и $b_{1}$, выполнены автоматически.) В свою очередь, для компонент векторов $\mathbf{p}$ и q мы получаем следующие 
уравнения:

$$
p_{j}+p_{j+g_{0}-1}=0, \quad q_{j}+q_{j+g_{0}-1}=0, \quad 2 \leqslant j \leqslant g_{0} .
$$
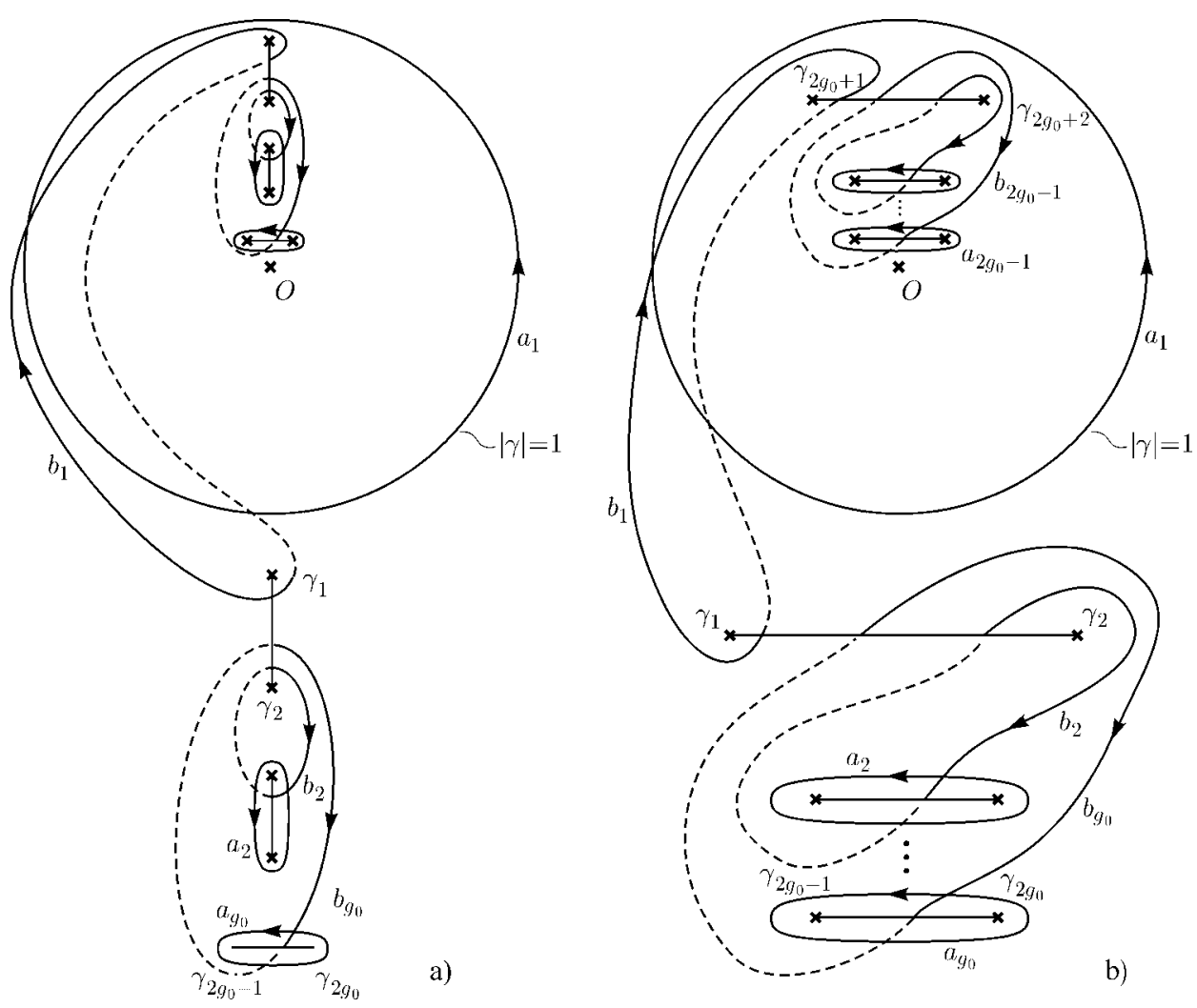

Рис. 2

Осталось понять, какие ограничения на $\mathbf{p}$ возникают из-за «условий вещественности» (2.12). Рассмотрим два случая по отдельности.

1. Неразделяющийся случай $(k \geqslant 1)$. Базисные циклы, изображенные на рис. 2а), ведут себя следующим образом под действием $\mu: \mu\left(a_{j}\right)=-a_{j}$ для любого $j, \mu\left(b_{1}\right)=b_{1}+2 a_{1}, \mu\left(b_{j}\right)=b_{j}$ при $2 \leqslant j \leqslant k, \mu\left(b_{j}\right)=b_{j}-a_{j}$ при $k+1 \leqslant j \leqslant g_{0}$ (поскольку мы уже предположили инвариантность $\mathscr{L}$ относительно $\sigma$, достаточно определить действие $\mu$ только на первых $g_{0}$ циклах $b_{j}$ ). Уравнение (2.12) налагает следующие условия на монодромии: $\bar{M}_{a_{j}}=M_{a_{j}}^{-1}$ для любого $j, \bar{M}_{b_{1}}=M_{b_{1}} M_{a_{1}}^{2}, \bar{M}_{b_{j}}=M_{b_{j}}$ при $2 \leqslant j \leqslant k, \bar{M}_{b_{j}}=M_{b_{j}} M_{a_{j}}^{-1}$ при $k+1 \leqslant j \leqslant g_{0}$, которые могут быть переписаны в терминах $\mathbf{p}$ и $\mathbf{q}$ :

$$
p_{j} \in \mathbb{R} \text { при любом } j, \quad \operatorname{Re} q_{1}=p_{1},
$$

$\operatorname{Re} q_{j}=0$ при $2 \leqslant j \leqslant k, \quad \operatorname{Re} q_{j}=-\frac{1}{2} p_{j}$ при $k+1 \leqslant j \leqslant g_{0}$.

2. Разделяющийся случай $(k=0)$. Выберем базисные циклы в соответствии с рис. $2 \mathrm{~b})$. Они преобразуются следующим образом под действием $\mu: \mu\left(a_{j}\right)=-a_{j}$ 
для любого $j, \mu\left(b_{1}\right)=b_{1}+\sum_{l=2}^{g_{0}}\left(a_{l+g_{0}-1}-a_{l}\right), \mu\left(b_{j}\right)=b_{j}-a_{j}+\sum_{l=1}^{g_{0}} a_{l}$ при $2 \leqslant j \leqslant g_{0}$, что налагает следующие условия на $\mathbf{p}$ и $\mathbf{q}:$

$$
\begin{array}{cl}
p_{j} \in \mathbb{R} \quad \text { для любого } j, & \operatorname{Re} q_{1}=-\frac{1}{2} \sum_{l=2}^{g_{0}} p_{l}, \\
\operatorname{Re} q_{j}=-\frac{1}{2} \sum_{l=1}^{g_{0}} p_{l}-\frac{p_{j}}{2} & \text { при } 2 \leqslant j \leqslant g_{0} .
\end{array}
$$

ТеОРемА 4.1. Пусть род кривой $\mathscr{L}$ нечетен, $g=2 g_{0}-1 ;$ nусть базисные изиклы на $\mathscr{L}$ выбраны, как показано на рис. $2 a)$, если кривая $\mathscr{L}$ относится $к$ неразделяющемуся типу, или как показано на рис. 2b), если кривая $\mathscr{L}$ относится к разделяющемуся типу. Возьмем два произвольных постоянных вектора $\mathbf{p}, \mathbf{q} \in \mathbb{C}^{2 g_{0}-1}$, удовлетворяющие условиям (4.3). Пусть, кроме того, $\mathbf{p} u$ q удовлетворяют условиям (4.4), если кривая $\mathscr{L}$ относится к неразделяющемуся типу, или условиям (4.5), если кривая $\mathscr{L}$ относится к разделяющемуся muпу. Определим функцию $\Psi(\gamma)$ выражениями (3.5)-(3.7). Тогда функция

$$
G(x, \rho) \equiv \Psi(x, \rho, \gamma=0)
$$

удовлетворяет уравнению Эрнста (2.10) и может быть представлена в ви$\partial e(2.9)$.

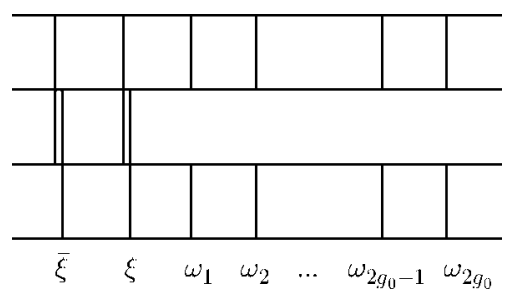

Рис. 3

С помощью (4.6) можно получить выражение для потенциала Эрнста в терминах тәта-функций, связанных с кривой $\mathscr{L}$. Это выражение может быть существенно упрощено, если использовать инвариантность $\mathscr{L}$ по отношению к инволюции $\sigma$ и перейти к спектральному параметру $w$ из (2.8). А именно, кривая $\mathscr{L}$ может быть реализована как четырехлистное накрытие над плоскостью $w$; ее диаграмма Гурвица изображена на рис. 3. При такой реализации инволюция $\sigma$ осуществляет перестановку листов $1 \leftrightarrow 3$ и $2 \leftrightarrow 4$, а инволюция * осуществляет перестановку листов $1 \leftrightarrow 2$ и $3 \leftrightarrow 4$. Введем новую кривую $\mathscr{L}_{0}$ рода $g_{0}$, определенную уравнением

$$
\nu^{2}=(w-\xi)(w-\bar{\xi}) \prod_{j=1}^{2 g_{0}}\left(w-w_{j}\right)
$$




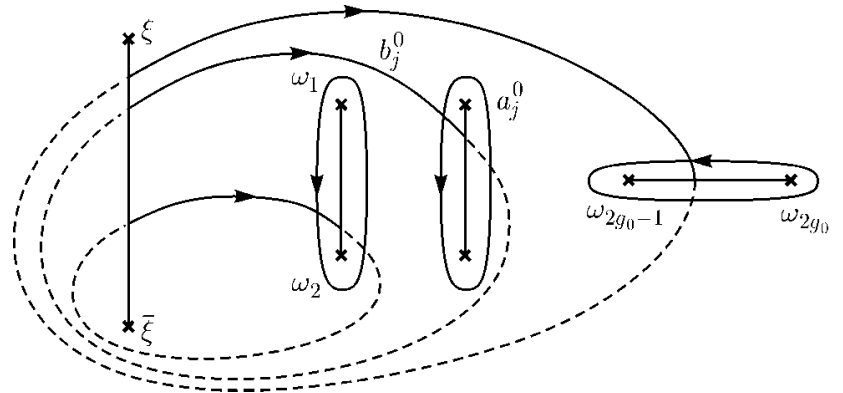

Рис. 4

Базис циклов $\left(a_{j}^{0}, b_{j}^{0}\right)$ на $\mathscr{L}_{0}$ выбран, как показано на рис. 4. Нормированный базис голоморфных 1-форм на $\mathscr{L}_{0}$ выглядит следующим образом:

$$
d V_{j}=\frac{1}{\nu} \sum_{k=1}^{g_{0}}\left(\mathscr{A}_{0}^{-1}\right)_{j k} w^{k-1} d w, \quad j=1, \ldots, g_{0},
$$

где

$$
\left(\mathscr{A}_{0}\right)_{k j} \equiv \oint_{a_{j}^{0}} \frac{w^{k-1} d w}{\nu}, \quad j, k=1, \ldots, g_{0} .
$$

Матрица $b$-периодов кривой $\mathscr{L}_{0}$ будет обозначаться через $\mathbf{B}_{0}$. Кривая $\mathscr{L}$ является двулистным неразветвленным накрытием П над $\mathscr{L}_{0}$; точки кривой $\mathscr{L}$, связанные инволюцией $\sigma$, проектируются в одну и ту же точку на $\mathscr{L}_{0}$. Антиинволюция $\mu$ действует на каждом листе кривой $\mathscr{L}_{0}$ как $w \rightarrow \bar{w}$. Наличие уравнения $(2.11)$ позволяет написать альтернативные формулы для функции $\Psi$ в терминах тэтафункций, связанных с кривой $\mathscr{L}_{0}$. Обозначим через $\Omega_{w}$ проекцию области $\Omega_{\gamma}$ на $w$-плоскость. Определим функцию $\Phi_{0}\left(w \in \Omega_{w}\right)$ по формуле

$$
\Phi_{0}\left(w \in \Omega_{w}\right)=\left(\begin{array}{ll}
\varphi_{0}(w) & \varphi_{0}\left(w^{*}\right) \\
\psi_{0}(w) & \psi_{0}\left(w^{*}\right)
\end{array}\right),
$$

где унаследованная с $\mathscr{L}$ инволюция $*$ переставляет листы кривой $\mathscr{L}_{0}$,

$$
\begin{array}{ll}
\varphi_{0}(w)=\Theta\left[\begin{array}{l}
\mathbf{r} \\
\mathbf{s}
\end{array}\right]\left(\left.V\right|_{\xi} ^{w} \mid \mathbf{B}_{0}\right), & \varphi_{0}\left(w^{*}\right)=-i \Theta\left[\begin{array}{l}
\mathbf{r} \\
\mathbf{s}
\end{array}\right]\left(-\left.V\right|_{\xi} ^{w} \mid \mathbf{B}_{0}\right), \\
\psi_{0}(w)=\Theta\left[\begin{array}{l}
\mathbf{r} \\
\mathbf{s}
\end{array}\right]\left(\left.V\right|_{\xi} ^{w}+\frac{1}{2} \mid \mathbf{B}_{0}\right), & \psi_{0}\left(w^{*}\right)=i \Theta\left[\begin{array}{l}
\mathbf{r} \\
\mathbf{s}
\end{array}\right]\left(-\left.V\right|_{\xi} ^{w}+\frac{1}{2} \mid \mathbf{B}_{0}\right),
\end{array}
$$

$w \in \Omega_{w}$, а постоянные векторы $\mathbf{r}, \mathbf{s} \in \mathbb{C}^{g_{0}}$ удовлетворяют условиям вещественности

$$
\mathbf{r} \in \mathbb{R}^{g_{0}}, \quad \operatorname{Re}_{j}=\sum_{l=1}^{g_{0}} \frac{\mathbf{r}_{l}}{2}, \quad j \leqslant k, \quad \operatorname{Re} \mathbf{s}_{j}=\sum_{l=1, l \neq j}^{g_{0}} \frac{\mathbf{r}_{l}}{2}, \quad j \geqslant k+1 .
$$

Базисные циклы кривой $\mathscr{L}_{0}$ преобразуются следующим образом под действием $\mu: \mu\left(a_{j}^{0}\right)=-a_{j}^{0}$ для любого $j, \mu\left(b_{j}^{0}\right)=b_{j}^{0}+\sum_{l=1}^{g_{0}} a_{l}^{0}, j \leqslant k, \mu\left(b_{j}^{0}\right)=b_{j}^{0}+\sum_{l \neq j} a_{l}^{0}$, $j>k$, что влечет за собой следующие соотношения на матрицу $b$-периодов кривой $\mathscr{L}_{0}: \operatorname{Re}\left(\mathbf{B}_{0}\right)_{j l}=-1 / 2, j \leqslant k, \operatorname{Re}\left(\mathbf{B}_{0}\right)_{j l}=-1 / 2+\delta_{j l} / 2, j>k$. Поэтому условия (4.12) могут быть эквивалентным образом переписаны в виде $\operatorname{Re}\left(\mathbf{B}_{0} \mathbf{r}+\mathbf{s}\right)=$ 0 , что, в частности, дает следующее соотношение между функциями $\varphi_{0}$ и $\psi_{0}$ : $\psi_{0}(P)=\overline{\varphi_{0}(\bar{P})}$. 
Теперь определим функцию

$$
\Psi_{0}\left(w \in \Omega_{w}\right) \equiv \sqrt{\frac{\operatorname{det} \Phi_{0}\left(\infty^{1}\right)}{\operatorname{det} \Phi_{0}(w)}} \Phi_{0}^{-1}\left(\infty^{1}\right) \Phi_{0}(w)
$$

и продолжим ее аналитически на все $X$; тем самым мы получаем функцию $\Psi_{0}(P \in X)$. Имеет место следующее утверждение:

Теорема 4.2. Определим функиию $\Psi(P \in X)$ формулами (3.5)-(3.7). Onределим также функцию $\Psi_{0}(P \in X)$ формулами (4.9), (4.10), (4.13). Пусть компоненты векторов $\mathbf{p}, \mathbf{q} \in \mathbb{C}^{2 g_{0}-1} u \mathbf{r}, \mathbf{s} \in \mathbb{C}^{g_{0}}$ связаны следующим образом:

$$
p_{1}=-\sum_{l=1}^{g_{0}} r_{l}, \quad q_{1}=-2 s_{1}, \quad p_{j}=r_{j}, \quad q_{j}=s_{j}-s_{1}, \quad 2 \leqslant j \leqslant g_{0}
$$

(соотношения (4.3) автоматически дают оставшиеся $g_{0}-1$ элементов из p и из q). Потребуем также, чтобы $\left[\begin{array}{l}\mathbf{r} \\ \mathbf{s}\end{array}\right]$ не совпадала ни с какой полуцелой характеристикой. Тогда функииии $\Psi$ и $\Psi_{0}$ совпадают: $\Psi(P \in X)=\Psi_{0}(P \in X)$.

ДокАЗАТЕЛЬСтво. Функции $\varphi_{0}$ и $\psi_{0}$ были выбраны таким образом, что аналитическое продолжение функции $\Psi_{0}$ с $\Omega_{w}$ на все $X$ не нарушает уравнения $(2.11)$. Принимая во внимание условие несовпадения $\left[\begin{array}{l}\mathbf{r} \\ \mathbf{s}\end{array}\right]$ ни с какой полуцелой характеристикой и совпадение условий нормировки функций $\Psi$ и $\Psi_{0}$ в точке $\gamma=\infty$, достаточно показать, что монодромии функций $\Psi$ и $\Psi_{0}$ вокруг первых $g_{0}$ пар базисных циклов кривой $\mathscr{L}$ совпадают. Базисные циклы на $\mathscr{L}$ и $\mathscr{L}_{0}$ связаны соотношениями

$$
\Pi\left(a_{1}\right)=-\left(a_{1}^{0}+\cdots+a_{g_{0}}^{0}\right), \quad \Pi\left(b_{1}\right)=-2 b_{1}^{0}, \quad \Pi\left(a_{j}\right)=a_{j}^{0}, \quad \Pi\left(b_{j}\right)=b_{j}^{0}-b_{1}^{0},
$$

которые дают следующие выражения для монодромий функции $\Psi_{0}$ вокруг $\left(a_{j}, b_{j}\right)$ :

$$
\begin{gathered}
M_{a_{1}}^{0}=\exp \left\{-2 \pi i \sum_{j=1}^{g_{0}} r_{j} \sigma_{3}\right\}, \quad M_{b_{1}}^{0}=\exp \left\{4 \pi i s_{1} \sigma_{3}\right\}, \\
M_{a_{j}}^{0}=\exp \left\{2 \pi r_{j} \sigma_{3}\right\}, \quad M_{b_{j}}^{0}=\exp \left\{2 \pi\left(s_{1}-s_{j}\right) \sigma_{3}\right\}, \quad 2 \leqslant j \leqslant g_{0} .
\end{gathered}
$$

Эти выражения совпадают с монодромиями кривой $\Psi$, которые даются формулами (3.8), если учесть соотношения (4.14) (уравнение (2.11) обеспечивает совпадение остальных монодромий вокруг циклов $\left.\left(a_{j}, b_{j}\right), g_{0}+1 \leqslant j \leqslant 2 g_{0}\right)$. [Заметим, что функции $\varphi_{0}$ и $\psi_{0}$ преобразуются по-разному (некоторые их монодромии отличаются знаком) при обходе вокруг базисных циклов кривой $\mathscr{L}_{0}$, но их аналитические продолжения на $\mathscr{L}$ преобразуются одинаковым образом при обходе вокруг базисных циклов кривой $\mathscr{L}$.]

Теорема 4.3. Рассмотрим два произвольных постоянных вектора $\mathbf{r}, \mathbf{s} \in \mathbb{C}^{g_{0}}$, удовлетворяющие условиям вещественности (4.12). Тогда функция

$$
\mathscr{E}(\xi, \bar{\xi})=\frac{\Theta\left[\begin{array}{l}
\mathbf{r} \\
\mathbf{s}
\end{array}\right]\left(\left.V\right|_{\xi} ^{\infty^{1}} \mid \mathbf{B}_{0}\right)}{\Theta\left[\begin{array}{l}
\mathbf{r} \\
\mathbf{s}
\end{array}\right]\left(\left.V\right|_{\xi} ^{\infty^{2}} \mid \mathbf{B}_{0}\right)}
$$

удовлетворяет уравнению Эрнста (2.5). Функция $\Psi$, определенная формулой (3.7) или (4.13), решает линейную систему (2.7), где матрица $G(\xi, \bar{\xi})$ задается формулой (2.9). 
ДоКАЗАТЕЛЬСтво. Нетривиальной частью является проверка формулы (4.17). А именно, матрицу $\Psi\left(w=\infty^{1}\right)$, задаваемую формулой (4.13), можно представить в виде $(2.9)$, где $\mathscr{E}=-i \varphi_{0}\left(\infty^{1}\right) / \varphi_{0}\left(\infty^{2}\right)$, что дает (4.17) после подстановки (4.10).

Теорема 4.4. Метрический коэффициент $F(\xi, \bar{\xi})$ (см. (2.6)), соответствующий потенциалу Эрнста (4.17), задается формулой

$$
F=\frac{2}{\operatorname{Re} \mathscr{E}} \operatorname{Im}\left\{\sum_{j=1}^{g_{0}}\left(\mathscr{A}_{0}^{-1}\right)_{g_{0} j} \frac{\partial}{\partial z_{j}} \ln \Theta\left[\begin{array}{l}
\mathbf{r} \\
\mathbf{s}
\end{array}\right]\left(\left.V\right|_{\xi} ^{\infty^{2}} \mid \mathbf{B}_{0}\right)\right\}
$$

с точностью до произвольной постоянной интегрирования, где $\mathscr{A}_{0}-$ матри-

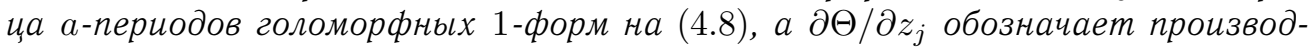
ную тэта-функиии по ее $j$-му аргументу.

ДокАЗАТЕЛЬСтво. Напишем следующее простое тождество:

$$
\left(\Psi^{-1} \Psi_{w^{-1}}\right)_{\xi} \equiv \Psi^{-1}\left(\Psi_{\xi} \Psi^{-1}\right)_{1 / w} \Psi .
$$

Принимая во внимание линейную систему (2.7), уравнение $(2.6)$ на $F$ и условие нормировки $\Psi(\infty)=I$, легко видеть, что $(1,2)$-матричный элемент в правой части этого тождества равен $\frac{1}{2} F_{\xi}$. Следовательно, $F=2\left(\Psi^{-1} \Psi_{w^{-1}}\right)_{12}(\gamma=\infty)$. Подстановка формул $(4.10),(4.11)$ и $(4.13)$ в эту формулу дает следующий результат:

$$
F=-\frac{2}{\operatorname{Re} \mathscr{E}} \operatorname{Im}\left\{\frac{d W_{0}(P)}{d(1 / w)}\left(w=\infty^{2}\right)\right\},
$$

где 1-форма $d W_{0}\left(P \in \mathscr{L}_{0}\right)$ задается выражением

$$
d W_{0}(P)=\sum_{j=1}^{g_{0}} d V_{j}(P) \frac{\partial}{\partial z_{j}} \ln \Theta\left[\begin{array}{l}
\mathbf{r} \\
\mathbf{s}
\end{array}\right]\left(V||_{\xi}^{P} \mid \mathbf{B}_{0}\right) .
$$

Используя формулу для базисных 1-форм $d V_{j}$, мы получаем (4.18).

Теорема 4.5. Метрический коэффициент $k(\xi, \bar{\xi})$, соответствующий потенциалу Эрнста (4.17), задается выражением

$$
e^{2 k}=\frac{\Theta\left[\begin{array}{l}
\mathbf{p} \\
\mathbf{q}
\end{array}\right](0 \mid \mathbf{B})}{\sqrt{\operatorname{det} \mathscr{A}_{0}}} \prod_{j=1}^{2 g_{0}}\left|w_{j}-\xi\right|^{-1 / 4}
$$

с точностью до произвольного постоянного множителя, где $\mathbf{B}$ - матрица b-периодов кривой $\mathscr{L}$, определяемой уравнением (3.1), матрица $\mathscr{A}_{0}$ определена уравнениями (4.8), а векторы $\mathbf{p}, \mathbf{q} \in \mathbb{C}^{2 g_{0}-1}$ связань с векторами $\mathbf{r}, \mathbf{s} \in \mathbb{C}^{g_{0}}$ формулами (4.14).

ДокАЗАТЕЛЬСТво. Нужно использовать совпадение функций $\Psi_{0}$ и $\Psi$ и подставить формулу для тау-функции системы Шлезингера (3.9) в соотношение (2.14), связывающее тау-функцию и коэффициент $e^{2 k}$ (в нашем случае $\operatorname{tr} A_{j}^{2}=1 / 8$ ). Кроме того, нужно использовать следующее соотношение между определителями матриц $a$-периодов голоморфных 1 -форм на кривых $\mathscr{L}$ и $\mathscr{L}_{0}: \operatorname{det} \mathscr{A}=$ const $\rho^{g_{0}^{2}} \operatorname{det} \mathscr{A}_{0}$, где константа не зависит от $(\xi, \bar{\xi})$. Доказательство этого равенства можно получить элементарными преобразованиями, используя разложение 
голоморфных 1-форм на $\mathscr{L}$ в комбинацию голоморфных 1 -форм на $\mathscr{L}_{0}$ и голоморфных 1-форм на не зависящей от $(\xi, \bar{\xi})$ гиперэллиптической кривой рода $g_{0}-1$, задаваемой уравнением $\delta^{2}=\prod_{j=1}^{2 g_{0}}\left(w-w_{j}\right)$. Для завершения доказательства формулы (4.19) остается использовать тождества

$\gamma^{-1} \gamma_{w}=\{(w-\xi)(w-\bar{\xi})\}^{-1 / 2}, \quad \prod_{j<k}\left(\gamma_{j}-\gamma_{k}\right)=\operatorname{const} \rho^{-4 g_{0}^{2}} \prod_{j=1}^{2 g_{0}}\left\{\left(w_{j}-\xi\right)\left(w_{j}-\bar{\xi}\right)\right\}^{1 / 2}$.

СледствиЕ 4.1. Метрический коэффициент (4.19) может быть выражен следующим образом через тэта-функциии кривой $\mathscr{L}_{0}$ :

$$
e^{2 k}=\frac{\Theta\left[\begin{array}{l}
\mathbf{r} \\
\mathbf{s}
\end{array}\right]\left(0 \mid \mathbf{B}_{0}\right) \Theta\left[\begin{array}{l}
\mathbf{r} \\
\mathbf{s}
\end{array}\right]\left(\frac{1}{2} \mathbf{e} \mid \mathbf{B}_{0}\right)}{\Theta\left(0 \mid \mathbf{B}_{0}\right) \Theta\left(\frac{1}{2} \mathbf{e} \mid \mathbf{B}_{0}\right)}
$$

гेe $\mathbf{e} \equiv(1, \ldots, 1)$.

ДокАЗАТЕЛЬСтво. Эта формула следует из (4.19), если использовать связь между тэта-функциями на $\mathscr{L}$ и $\mathscr{L}_{0}[15$, р. 64] и тот факт, что $k=0$ при $\mathbf{r}=\mathbf{s}=0$.

ЗАмечАНИЕ 4.1. Вырождение $w_{2 j+1}, w_{2 j+1} \rightarrow \kappa_{j} \in \mathbb{R}$ кривой $\mathscr{L}_{0}$ дает при подходящем выборе $\mathbf{r}$ и $\mathbf{s}$ все семейство многосолитонных решений. В частности, чтобы получить решение Керра-НУТ, нужно взять $g_{0}=2, r_{j}=1 / 2, s_{j}=1 / 4+$ $i \alpha_{j}, \alpha_{j} \in \mathbb{R}$. Тогда формула (4.17) дает [4]

$$
\mathscr{E}=\frac{1-\Gamma}{1+\Gamma}, \quad \Gamma^{-1}=\frac{i\left(a_{1}-a_{2}\right)}{1-a_{1} a_{2}+i\left(a_{1}+a_{2}\right)} \mathscr{X}^{+}+\frac{1+a_{1} a_{2}}{1-a_{1} a_{2}+i\left(a_{1}+a_{2}\right)} \mathscr{X}^{-},
$$

где $a_{j}=e^{-2 \pi i \alpha_{j}}$ и сплющенные эллипсоидальные координаты задаются формулами $\mathscr{X}^{ \pm}=\left(\kappa_{1}-\kappa_{2}\right)^{-1}\left\{\sqrt{\left(\kappa_{1}-\xi\right)\left(\kappa_{1}-\bar{\xi}\right)} \pm \sqrt{\left(\kappa_{2}-\xi\right)\left(\kappa_{2}-\bar{\xi}\right)}\right\}$.

В работе [4] были построены тэта-функциональные решения уравнения Эрнста (в обозначениях, введенных в $[6,9]$ )

$$
\mathscr{E}=\frac{\Theta\left(\left.V\right|_{\xi} ^{\infty^{1}}+B_{W} \mid \mathbf{B}_{0}\right)}{\Theta\left(\left.V\right|_{\xi} ^{\infty^{2}}+B_{W} \mid \mathbf{B}_{0}\right)} \exp \left\{\left.W\right|_{\infty^{2}} ^{\infty^{1}}\right\}
$$

где $d W(P)-$ произвольная 1 -форма на $\mathscr{L}_{0}$, удовлетворяющая следующим условиям:

1. $d W$ является произвольной конечной, бесконечной или непрерывной линейной комбинацией с не зависящими от $(\xi, \bar{\xi})$ коэффициентами абелевых дифференциалов второго и третьего рода, полюсы и сингулярные части которых не зависят от $(\xi, \bar{\xi})$.

2. (Условия вещественности.) $d W(\bar{P})=\overline{d W(P)}$ и $2 \pi i B_{W} \in \mathbb{R}^{g_{0}}$, где вектор $2 \pi i B_{W}$ - это вектор $b$-периодов дифференциала $d W: 2 \pi i\left(B_{W}\right)_{j}=\oint_{b_{j}} d W(P)$.

3. (Условия нормировки.) $\oint_{a_{j}} d W=0$ для любого $j$.

Для прояснения роли дифференциала $d W$ рассмотрим сначала простейший случай $g=0$, когда потенциал Эрнста (4.20) становится вещественным,

$$
\ln \mathscr{E}=\left.W\right|_{\infty^{2}} ^{\infty^{1}}
$$


и уравнение Эрнста сводится к уравнению Эйлера-Дарбу $\left(\partial_{\rho}^{2}+\rho^{-1} \partial_{\rho}+\partial_{z}^{2}\right) \ln \mathscr{E}$ $=0$. Естественно возникает вопрос о возможности представления общего решения уравнения Эйлера-Дарбу, инвариантного по отношению к изменению знака $\rho$, в форме (4.21). Ответ оказывается положительным. А именно, фиксируем некоторую область $D$ в $(\rho, z)$-плоскости, симметричную относительно отражения $\rho \rightarrow-\rho$ (область $D$ может захватывать бесконечно удаленную точку с асимптотическим граничным условием $\ln \mathscr{E} \rightarrow 0$ при $\rho, z \rightarrow \infty)$. Тогда общее несингулярное решение уравнения Эйлера-Дарбу в области $D$, симметричное по отношению к тому же отражению, может быть записано в виде интеграла вдоль границы $\partial D$ :

$$
\ln \mathscr{E}=\int_{\partial D} \frac{f(\kappa) d \kappa}{\{(\kappa-\xi)(\kappa-\bar{\xi})\}^{1 / 2}},
$$

где $f(\kappa)$ - произвольная функция, определенная на $\partial D$ и удовлетворяющая условию вещественности $f(\bar{\kappa})=\overline{f(\kappa)}$. Решение $(4.22)$ может быть записано в виде (4.21), где $d W(w)=\frac{1}{2} \oint_{\partial D} f(\kappa)\left(d W_{\kappa}(w)-d W_{\kappa^{*}}(w)\right) d \kappa$ и $d W_{\kappa}(w)-$ дифференциал второго рода на кривой $\mathscr{L}_{0}$ с $g_{0}=0$. Дифференциал $d W_{\kappa}(w)$ имеет единственный полюс второго порядка в точке $w=\kappa$ со следующим асимптотическим разложением в его окрестности: $d W_{\kappa}(w)=\left((w-\kappa)^{-2}+O(1)\right) d w$ при $w \rightarrow \kappa$. Поскольку $g_{0}=0$, мы можем записать $d W_{\kappa}(w)$ явным образом:

$$
d W_{\kappa}(w)=\frac{\gamma^{\prime}(\kappa) \gamma^{\prime}(w) d w}{(\gamma(w)-\gamma(\kappa))^{2}}
$$

и, следовательно,

$$
\int_{\infty^{1}}^{\infty^{2}} d W_{\kappa}=\left.\partial_{\kappa}(\ln \gamma)\right|_{\gamma=\infty} ^{\gamma=0}=\{(\kappa-\xi)(\kappa-\bar{\xi})\}^{-1 / 2},
$$

что показывает совпадение решений (4.21) и (4.22). Интегрирование по частям дает следующее альтернативное представление для $d W$ :

$$
d W(w)=\oint_{\partial D} h(\kappa) d W_{\kappa \kappa^{*}}(w) d \kappa,
$$

где $h(\kappa)=-f^{\prime}(\kappa) / 2$, а $d W_{\kappa \kappa^{*}}(w)-$ стандартный дифференциал третьего рода с полюсами в точках $\kappa$ и $\kappa^{*}$, задаваемый формулой

$$
d W_{\kappa \kappa^{*}}(w)=\left(\frac{\gamma^{\prime}(w)}{\gamma(w)-\gamma(\kappa)}-\frac{\gamma^{\prime}(w)}{\gamma(w)-\gamma^{-1}(\kappa)}\right) d w .
$$

Интегральное представление (4.25) дифференциала $d W$ соответствует проинтегрированному по частям соотношению $(4.22): \ln \mathscr{E}=2 \int_{\partial D} h(\kappa) \ln \gamma(\kappa, \xi, \bar{\xi}) d \kappa$. Повторяя интегрирование по частям, мы можем представить $d W$ в виде контурного интеграла по $\kappa$ от мероморфных дифференциалов, имеющих полюс произвольного фиксированного порядка при $w=\kappa$ и $w=\kappa^{*}$. Таким образом, с точки зрения локальных решений не все 1-формы $d W$ в (4.21) являются независимыми, и для получения общего локального статического решения достаточно ограничиться формами вида (4.25).

Рассмотрим форму $d W$ в случае $g_{0}>0$. Обозначим через $d W_{Q R}(P)$ дифференциал третьего рода на $\mathscr{L}_{0}$, имеющий полюсы первого порядка в точках $Q$ и $R$ с вычетами +1 и -1 соответственно и удовлетворяющий условиям нормировки 
$\oint_{a_{j}} d W_{Q R}(P)=0$ для любого $j$. Его вектор $b$-периодов $2 \pi i\left(B_{Q R}\right)_{j} \equiv \oint_{b_{j}} d W_{Q R}$ может быть выражен через базисные формы $d V_{j}$ :

$$
\left(B_{Q R}\right)_{j}=\int_{R}^{Q} d V_{j}
$$

При $g_{0} \geqslant 1$, как и в случае $g_{0}=0$, одна и та же форма $d W\left(P \in \mathscr{L}_{0}\right)$ может быть представлена разными способами в виде контурного интеграла от элементарных 1-форм. Для наших целей будет удобно использовать представление, являющееся обобщением представления (4.25) на случай $g_{0} \geqslant 1$ :

$$
d W=\oint_{\partial D} h(\kappa) d W_{\kappa \kappa^{*}} d \kappa .
$$

Соответствующий этому представлению вектор $b$-периодов дается формулой $\left(B_{W}\right)_{j}=$ $\left.2 \oint_{\partial D} h(\kappa) V_{j}\right|_{\xi} ^{\kappa} d \kappa$.

Далее мы собираемся установить связь между решениями (4.17) и (4.20). На первый взгляд кажется, что решения (4.17) содержат меньшее число параметров, однако оказывается, что на самом деле оба класса решений совпадают.

ЗАмечАниЕ 4.2. Класс мероморфных дифференциалов $d W$, удовлетворяющих условиям 1-3, тесно связан с классом дифференциалов, которые были введены Кричевером [17] в конструкции точных решений уравнений Уизема, описывающих специального вида деформации конечнозонных решений уравнений типа Кортевега-де Фриза ${ }^{1}$. Такого же типа объекты возникают в некоторых примеpax фробениусовых многообразий [18], связанных с пространствами Гурвица. Поскольку, как известно, уравнения, описывающие фробениусовы многообразия, сводятся, как и уравнение Эрнста, к уравнениям изомонодромных деформаций, возникает естественный вопрос о соотношении соответствующих классов алгебро-геометрических решений. Поверхностный анализ не обнаруживает простой связи между двумя ситуациями в общем случае; однако мы надеемся, что такого рода связь может проявиться при более детальном рассмотрении.

Чтобы понять, как решения (4.17) возникают из решений (4.20), мы сначала обсудим, как голоморфные дифференциалы на $\mathscr{L}_{0}$ возникают в пределе дифференциалов третьего рода. А именно, рассмотрим дифференциал $d W_{Q R}(P)$ в фундаментальном многоугольнике $\widehat{\mathscr{L}}_{0}$, граница которого состоит из контуров $a_{j}^{0^{+}}$, $b_{j}^{0^{+}}, a_{j}^{0^{-}}, b_{j}^{0^{-}}$. Очевидно, что в пределе $Q \rightarrow R$ дифференциал $d W_{Q R}$ должен превращаться в некоторый голоморфный дифференциал. Самый наивный способ перейти к этому пределу - это предположить, что $Q \rightarrow R$ внутри $\widehat{\mathscr{L}}_{0}$; тогда $d W_{Q R} \rightarrow 0$ и $B_{Q R} \rightarrow 0$. Однако этот предел может быть вычислен и двумя нетривиальными способами:

1. Устремим $Q$ к какой-нибудь граничной точке многоугольника $\widehat{\mathscr{L}}_{0}$, скажем, к точке, лежащей на контуре $b_{j}^{0^{+}}$, и пусть $R$ одновременно стремится к той же точке кривой $\mathscr{L}_{0}$, лежащей на контуре $b_{j}^{0-}$. Этот предельный переход не влияет на условие нормировки по $а$-циклам, и, следовательно, $d W_{Q R} \rightarrow 0$ при $Q \rightarrow$ $R \in b_{j}^{0}$. Однако вектор $b$-периодов дифференциала $d W_{Q R}$ не обращается в нуль даже в пределе: в силу (4.27) мы видим, что $\left(B_{Q R}\right)_{k} \rightarrow-\delta_{j k}$ при $Q \rightarrow R \in b_{j}^{0}$.

\footnotetext{
${ }^{1}$ Мы благодарим рецензента, который обратил наше внимание на данное обстоятельство.
} 
2. Аналогично мы можем перейти к пределу, предполагая, что $Q$ стремится к некоторой точке, лежащей на контуре $a_{j}^{0^{+}}$, а $R$ стремится к соответствующей точке контура $a_{j}^{0-}$. Поскольку полюсы дифференциала $d W_{Q R}$ встречаются в точности на контуре $a_{j}^{0}$, мы видим, что интеграл $\oint_{a_{j}^{0}} d W_{Q R}$ в пределе не обращается в нуль. Поскольку $\oint_{a_{k}^{0}} d W_{Q R}$ по-прежнему равен нулю при $k \neq j$, мы заключаем, что в нашем пределе $d W_{Q R}$ становится пропорционален $d V_{j}$. Чтобы вычислить коэффициент пропорциональности, мы вычислим предельное значение вектора $b$ периодов формы $d W_{Q R}$ в соответствии с (4.27) и получим, что $B_{Q R} \rightarrow \mathbf{B}_{0 j}$ при $Q \rightarrow R \in a_{j}^{0}$, где $\mathbf{B}_{0 j}$ обозначает $j$-й столбец матрицы $b$-периодов. Следовательно, $d W_{Q R} \rightarrow 2 \pi i d V_{j}$ при $Q \rightarrow R \in a_{j}^{0}$.

Теперь выберем форму $d W$ в решении (4.20) в следующем виде:

$$
d W=\sum_{j=1}^{g_{0}}\left(r_{j} d W_{Q_{j} R_{j}}-s_{j} d W_{\widetilde{Q}_{j} \widetilde{R}_{j}}\right)
$$

с постоянными векторами $\mathbf{r}, \mathbf{s} \in \mathbb{C}^{g_{0}}$, и вычислим пределы $Q_{j} \rightarrow R_{j} \in a_{j}^{0}, \widetilde{Q}_{j} \rightarrow$ $\widetilde{R}_{j} \in b_{j}^{0}$, как описано в пп. 1 и 2 соответственно. Тогда, в соответствии с только что полученными формулами, мы имеем $B_{W} \rightarrow \mathbf{B}_{0} \mathbf{r}+\mathbf{s},\left.\left.W\right|_{\infty^{2}} ^{\infty^{1}} \rightarrow \sum_{j=1}^{g_{0}} r_{j} V_{j}\right|_{\infty^{2}} ^{\infty^{1}}$. Условие вещественности $d W(\bar{P})=\overline{d W(P)}$ налагает следующее ограничение на константы $\mathbf{r}$ и $\mathbf{s}: \operatorname{Re}\left\{\mathbf{B}_{0} \mathbf{r}+\mathbf{s}\right\}=0$. Таким образом, решение (4.20) принимает в нашем пределе следующий вид:

$$
\mathscr{E}=\frac{\Theta\left(\left.V\right|_{\xi} ^{\infty^{1}}+\mathbf{B}_{0} \mathbf{r}+\mathbf{s} \mid \mathbf{B}_{0}\right)}{\Theta\left(\left.V\right|_{\xi} ^{\infty^{2}}+\mathbf{B}_{0} \mathbf{r}+\mathbf{s} \mid \mathbf{B}_{0}\right)} \exp \left\{\left.\sum_{j=1}^{g_{0}} r_{j} V_{j}\right|_{\infty^{2}} ^{\infty^{1}}\right\},
$$

что совпадает с (4.17), если учесть, что тэта-функция с характеристиками есть обычная тэта-функция от сдвинутого аргумента, домноженная на некоторый экспоненциальный множитель.

Теперь опишем несколько более нетривиальную процедуру перехода от решения $(4.17)$ к (4.20), (4.28). Рассмотрим решение (4.17), в котором кривая $\mathscr{L}_{0}$ заменена на кривую $\mathscr{L}_{1}$ рода $g_{1}=g_{0}+n$, задаваемую уравнением

$$
\nu^{2}=(w-\xi)(w-\bar{\xi}) \prod_{j=1}^{2 g_{0}+2 n}\left(w-w_{j}\right),
$$

и выберем векторы $\mathbf{r}, \mathbf{s} \in \mathbb{C}^{g_{0}+n}$ следующим образом: $\mathbf{s}=0, r_{j}=0,1 \leqslant j \leqslant g_{0}$, $r_{g_{0}+j}=h_{j} \in \mathbb{R}, 1 \leqslant j \leqslant n$. Без потери общности мы будем считать, что $h_{j} \in[0,1]$. Матрицу $b$-периодов кривой $\mathscr{L}_{1}$ мы обозначим через $\mathbf{B}_{1}$, а базис голоморфных 1 -форм - через $d V_{1}, \ldots, d V_{g_{0}+n}$. Напишем решение (4.17), построенное по этим данным:

$$
\mathscr{E}=\frac{\Theta\left[\begin{array}{l}
\mathbf{r} \\
0
\end{array}\right]\left(\left.V\right|_{\xi} ^{\infty^{1}} \mid \mathbf{B}_{1}\right)}{\Theta\left[\begin{array}{l}
\mathbf{r} \\
0
\end{array}\right]\left(\left.V\right|_{\xi} ^{\infty^{2}} \mid \mathbf{B}_{1}\right)},
$$

и совершим предельный переход $w_{2 g_{0}+2 j-1}, w_{2 g_{0}+2 j} \rightarrow \kappa_{j} \in \mathbb{R}$. Кривая $\mathscr{L}_{1}$ превращается в кривую $\mathscr{L}_{0}$ с двойными точками в $\kappa_{j}, j=1, \ldots, n$. Канонический базис голоморфных 1-форм на $\mathscr{L}_{1}$ превращается в $d V_{1}, \ldots, d V_{g_{0}}, \frac{1}{2 \pi i} d W_{\kappa_{1} \kappa_{1}^{*}}, \ldots$, $\frac{1}{2 \pi i} d W_{\kappa_{n} \kappa_{n}^{*}}$, где $d V_{1}, \ldots, d V_{g_{0}}-$ канонический базис голоморфных 1 -форм на 
$\mathscr{L}_{0}$ и $d W_{\kappa_{j} \kappa_{j}^{*}}-$ нормированные дифференциалы 3 -го рода на $\mathscr{L}_{0}$. Следовательно, матрица $b$-периодов кривой $\mathscr{L}_{1}$ ведет себя в пределе следующим образом: $\left(\mathbf{B}_{1}\right)_{j k}=\left(\mathbf{B}_{0}\right)_{j k}+o(1), 1 \leqslant j, k \leqslant g_{0},\left(\mathbf{B}_{1}\right)_{j, k+g_{0}}=\left.2 V_{j}\right|_{\xi} ^{\kappa_{k}}+o(1), 1 \leqslant j \leqslant g_{0}$, $1 \leqslant k \leqslant n,\left(\mathbf{B}_{1}\right)_{g_{0}+j, g_{0}+j}=-\frac{1}{\pi i} \ln \left|w_{2 g_{0}+2 j-1}-w_{2 g_{0}+2 j}\right|+O(1), 1 \leqslant j \leqslant n$, $\left(\mathbf{B}_{1}\right)_{g_{0}+k, g_{0}+j}=O(1), 1 \leqslant j \neq k \leqslant n$. Подставляя эти соотношения в определение тэта-функции, мы получаем в нашем пределе следующее выражение для решения (4.30), в котором все объекты строятся по кривой $\mathscr{L}_{0}$ :

$$
\mathscr{E}=\frac{\Theta\left(\left.V\right|_{\xi} ^{\infty^{1}}+\left.2 \sum_{j=1}^{n} h_{j} V\right|_{\xi} ^{\kappa_{j}} \mid \mathbf{B}_{0}\right)}{\Theta\left(\left.V\right|_{\xi} ^{\infty^{2}}+\left.2 \sum_{j=1}^{n} h_{j} V\right|_{\xi} ^{\kappa_{j}} \mid \mathbf{B}_{0}\right)} \exp \left\{\left.\sum_{j=1}^{n} h_{j} W_{\kappa_{j} \kappa_{j}^{*}}\right|_{\infty^{2}} ^{\infty^{1}}\right\} .
$$

ЗАмЕчАНИЕ 4.3. Предположение $\kappa_{j} \in \mathbb{R}, h_{j} \in \mathbb{R}$ было сделано из соображений удобства; мы можем также предположить наличие в (4.31) сопряженных пар $\kappa_{j}=$ $\bar{\kappa}_{l}, h_{j}=\bar{h}_{l}$, которые возникают из склеивания двух «вертикальных» разрезов.

Мы можем вычислить непрерывный предел в $(4.31)$, распределяя точки $\kappa_{j}$ вдоль произвольного контура $\partial D$ с произвольной (скажем, непрерывной) мерой $h(\kappa)$, удовлетворяющей условиям вещественности $h(\bar{\kappa})=\overline{h(\kappa)}$. Тогда решение (4.31) принимает вид

$$
\mathscr{E}=\frac{\Theta\left(\left.V\right|_{\xi} ^{\infty^{1}}+2 \oint_{\partial D} h(\kappa)\left\{\left.V\right|_{\xi} ^{\kappa}\right\} d \kappa \mid \mathbf{B}_{0}\right)}{\Theta\left(\left.V\right|_{\xi} ^{\infty^{2}}+2 \oint_{\partial D} h(\kappa)\left\{\left.V\right|_{\xi} ^{\kappa}\right\} d \kappa \mid \mathbf{B}_{0}\right)} \exp \left\{\oint_{\partial D} h(\kappa)\left\{\left.W_{\kappa \kappa^{*}}\right|_{\infty^{2}} ^{\infty^{1}}\right\} d \kappa\right\},
$$

который совпадает с $(4.20)$, (4.28). Переходя к непрерывному пределу в формулах для метрических коэффициентов, соответствующих потенциалу Эрнста (4.31), можно получить выражения для функций $e^{2 k}$ и $F$, соответствующих потенциалу Эрнста (4.32).

Теорема 4.6. Коэффицииен F метрики (2.4), соответствующий решению (4.32) уравнения Эрнста, дается следующим выражением:

$$
\begin{array}{r}
F=\frac{2}{\operatorname{Re} \mathscr{E}} \operatorname{Im}\left\{\sum_{j=1}^{g_{0}}\left(\mathscr{A}_{0}^{-1}\right)_{g_{0} j} \frac{\partial}{\partial z_{j}} \ln \Theta\left(\left.V\right|_{\xi} ^{\infty^{2}}+2 \oint_{\partial D} h(\kappa)\left\{V||_{\xi}^{\kappa}\right\} d \kappa \mid \mathbf{B}_{0}\right)\right. \\
\left.+\oint_{\partial D} h(\kappa) d \kappa \frac{d W_{\kappa \kappa^{*}}}{d w^{-1}}\left(\infty^{2}\right)\right\},
\end{array}
$$

где все объекты строятся по кривой $\mathscr{L}_{0}$. Соответствующий метрический коэффициент $e^{2 k}$ задается выражением ${ }^{1}$

$$
\begin{aligned}
e^{2 k}=\frac{\Theta\left(2 \oint_{\partial D} H(\kappa) d V(\kappa) \mid \mathbf{B}_{0}\right) \Theta\left(2 \oint_{\partial D} H(\kappa) d V(\kappa)+\frac{1}{2} \mathbf{e} \mid \mathbf{B}_{0}\right)}{\Theta\left(0 \mid \mathbf{B}_{0}\right) \Theta\left(\frac{1}{2} \mathbf{e} \mid \mathbf{B}_{0}\right)} \\
\quad \times \exp \left\{2 \oint_{\partial D} \oint_{\partial D} h(\kappa) h(\tilde{\kappa}) \ln \frac{\Theta[S]\left(V(\tilde{\kappa})-V(\kappa) \mid \mathbf{B}_{0}\right)}{\kappa-\tilde{\kappa}} d \kappa d \tilde{\kappa}\right\},
\end{aligned}
$$

\footnotetext{
${ }^{1}$ Как нам сообщили Майнель и Нойгебауэр, похожая формула была получена ими для частного случая решений рода 2 .
} 
где $H(\kappa)$ - первообразная от функции $h: h(\kappa)=d H(\kappa) / d \kappa$, a $[S]-$ произвольная несингулярная нечетная характеристика на $\mathscr{L}_{0}$.

БлАгодАРНОсть. Мы благодарим Кристиана Клейна, при участии которого последняя формула была выведена из (4.19).

\section{ЛИТЕРАТУРА}

1. Белинский B. A., Захаров B. E. Интегрирование уравнений Эйнштейна методом обратной задачи рассеяния и вычисление точных солитонных решений. ЖЭТФ, 48, 985 (1978).

2. Maison $D$. Are the stationary, axially symmetric Einstein equations completely integrable? Phys. Rev. Lett., 41, 521 (1978).

3. Bianchi L. Lezioni di geometria differenziale. Pisa, 1909.

4. Короткин Д. А. Конечнозонные решения стационарного аксиально-симметричного уравнения Эйнштейна в вакууме. ТМФ, 77, № 1, 25-41 (1988).

5. Короткин Д. А., Матвеев В. Б. Алгеброгеометрические решения уравнения гравитации. Алгебра и анализ, 1, вып. 2, 77-102 (1990).

6. Korotkin D. Elliptic solutions of stationary axisymmetric Einstein equation. Class. Quantum Gravity, 10, 2587-2613 (1993).

7. Neugebauer G., Meinel $R$. General relativistic gravitational field of the rigidly rotating disk of dust: solution in terms of ultraelliptic functions. Phys. Rev. Lett., 75, 3046-3048 (1995).

8. Neugebauer G., Meinel R. Solutions to Einstein's field equation related to Jacobi inversion problem. Phys. Lett. A, 210, 160 (1996).

9. Korotkin D. Some remarks on finite-gap solutions of Ernst equation. Phys. Lett. A, 229, 195-199 (1997).

10. Klein C., Richter O. Explicit solutions of Riemann-Hilbert problem for the Ernst equation. Phys. Rev. D, 57, 857-862 (1998).

11. Зверович Е. И. Граничные задачи в теории аналитических функций в гёльдеровских классах на римановых поверхностях. УМН, 26, 117-192 (1971).

12. Schlesinger $L$. Über eine Klasse von Differentialsystemen beliebiger Ordnung mit festen kritischen Punkten. J. Reine Angew. Math., 141, 96-145 (1912).

13. Kitaev A., Korotkin D. On solutions of the Schlesinger equations in terms of thetafunctions. Intern. Math. Research Notices, 17, 877-906 (1998).

14. Korotkin D., Nicolai $H$. Isomonodromic quantization of dimensionally-reduced gravity. Nuclear Phys. B, 475, 397-439 (1996).

15. Fay John D. Theta-functions on Riemann surfaces. Lect. Notes in Math., vol. 352, Springer-Verlag, Berlin, 1973.

16. Jimbo M., Miwa T., Ueno K. Monodromy preserving deformation of linear ordinary differential equations with rational coefficients I. Phys. D, 2, 306-352 (1981).

17. Кричевер И. М. Метод усреднения для двумерных «интегрируемых» уравнений. Функц. анализ и его прил., 22, вып. 3, 37-52 (1988).

18. Dubrovin B. Geometry of 2D topological field theories. In: Integrable Systems and Quantum Groups, Lect. Notes in Math., vol. 1620 (Francaviglia M. and Greco S., eds.), Springer-Verlag, 1996, pp. 120-348.

Concordia University (Montreal)

Поступило в редакцию

Петербургское отделение математического 26 апреля 1999 г.

института им. В. А. Стеклова РАН

Dijon University 